\title{
Relationship between Serotonergic Dysfunction Based on Loudness Dependence of Auditory-Evoked Potentials and Suicide in Patients with Major Depressive Disorder
}

\author{
Young-Min Park \\ Department of Psychiatry, Inje University College of Medicine, Ilsan Paik Hospital, Goyang, Republic of Korea
}

The relationship between suicidality and the loudness dependence of auditory-evoked potentials (LDAEP) remains controversial. This article reviews the literature related to the LDAEP and suicide in patients with major depressive disorder, and suggests future research directions. Serotonergic dysfunction in suicidality seems to be more complicated than was originally thought. Studies of suicide based on the LDAEP have produced controversial results, but it is possible that these are due to differences in study designs and the smallness of samples. For example, some studies have evaluated suicide ideation and the LDAEP, while others have evaluated suicide attempts and the LDAEP. Furthermore, some of the latter studies enrolled acute suicide attempters, while others enrolled those with the history of previous suicide attempts, irrespective of whether these were acute or chronic. Thus, a more robust study design is needed in future studies, for example by evaluating the LDAEP immediately after a suicide attempt rather than in those with a history of suicide attempts and suicide ideation in order to reduce bias. Moreover, genuine suicide attempt, self-injurious behaviors, and faked suicide attempt need to be discriminated in the future.

Key Words LDAEP, Major depressive disorder, Suicide, Suicide attempt.

\section{INTRODUCTION}

Individuals who have committed or attempted suicide are thought to have some biological abnormalities, but the biological markers for suicide remain unclear. ${ }^{1}$ Most of the available evidence indicates that reduced serotonin plays an essential role in the pathogenesis of suicide. ${ }^{2}$ Early studies showed that the cerebrospinal fluid (CSF) levels of 5-hydroxyindoleacetic acid (5-HIAA), which is the metabolite of serotonin and serotonin, were reduced in depressive patients and suicide victims. ${ }^{3,4}$ In addition, suicide attempters show not only a significant reduction of serotonin binding to its transporter in the ventral prefrontal cortex, ${ }^{5}$ but also a significant reduction of serotonin binding to the 5-hydroxytryptamine $2 \mathrm{~A}$ receptor. ${ }^{6,7}$ Some genetic studies have found a relationship between serotonin-related genes and suicide., ${ }^{8,9}$ The prolactin

Received: May 8, 2015 Revised: June 18, 2015

Accepted: June 18, 2015 Available online: September 30, 2015

$\triangle$ Correspondence: Young-Min Park, MD, PhD

Department of Neuropsychiatry, Inje University College of Medicine, Ilsan Paik Hospital, 170 Juhwa-ro, Ilsanseo-gu, Goyang 10380, Republic of Korea Tel: +82-31-910-7260, Fax: +82-31-910-7268, E-mail: medipark@hanmail.net

(a) This is an Open Access article distributed under the terms of the Creative Commons Attribution Non-Commercial License (http://creativecommons.org/licenses/bync/3.0) which permits unrestricted non-commercial use, distribution, and reproduction in any medium, provided the original work is properly cited. response in the fenfluramine test was found to be smaller in depressive suicide attempters than in depressive non-suicideattempters and normal healthy controls. ${ }^{10}$ This means that serotonin activity was lower in depressive suicide attempters, since fenfluramine facilitates the release of serotonin that leads to an increase in prolactin secretion.

Serotoninergic activity may help to predict the risk of suicide attempts in depressive patients. However, the methods, such as taking a CSF sample or applying the fenfluramine challenge test, are invasive and/or complicated for psychiatric outpatients. Therefore, easy and noninvasive methods of measuring the central serotonergic activity are needed. Both preclinical and animal research indicates that the loudness dependence of auditory-evoked potentials (LDAEP) is a reliable indicator of central serotonergic activity. ${ }^{11,12}$ The LDAEP has been identified as being inversely associated with central serotonergic activity, with a small LDAEP reflecting strong serotonergic neurotransmission. ${ }^{12-16}$ Patients with major depression who have a larger LDAEP before taking medication exhibited a more favorable response to serotonergic antidepressants. ${ }^{15}$ In addition, a small pretreatment LDAEP was related to unresponsiveness and severe adverse effects in response to selective serotonin-reuptake inhibitors. ${ }^{17,18}$ Thus, measuring the 
LDAEP appears to provide useful clinical information for predicting treatment responses relative to central serotonergic activity.

Many studies have measured evoked potentials in patients with major depressive disorder (MDD), but only a few have focused on electrophysiological aberrance associated with suicide attempts. A recent study yielded electrophysiological evidence that the serotonergic activity was even lower (i.e., a larger LDAEP) in unmedicated depressive suicide attempters than in their depressive counterparts who did not attempt suicide. ${ }^{19}$ In contrast, Uhl and colleagues reported that patients with a history of suicide attempts exhibit a small LDAEP; however, the drug washout period allowed in that study was only 3 days. $^{20}$

The relationship between suicidality and the LDAEP therefore remains controversial. In this article we review the literature related to the LDAEP and suicide in patients with MDD, and suggests future research directions.

\section{LDAEP AND SUICIDE IDEATION IN PATIENTS WITH MAJOR DEPRESSIVE DISORDER}

A previous study found that patients who had acute suicidal ideas, as found in item 3 of the Hamilton Depression Rating Scale (HAMD), were mainly present in the group with strong intensity dependences, when 22 patients with MDD were divided into 2 groups based on the median LDAEP value. ${ }^{21}$ Another study found that the LDAEP did not differ significantly between 73 depressive outpatients with or without suicide ideation, as dichotomized based on the score for HAMD item 3 or Beck Depression Inventory (BDI) item 9.22 A recent study investigating the LDAEP and suicidality related to the subtype of MDD found a significant negative correlation between the LDAEP and the Beck Scale for Suicide Ideation (BSS) score in the atypical depression group $(n=53)$, but no significant correlations between the LDAEP and scores on psychological scales in the non-atypical depression group $(n=68){ }^{23}$ In addition, the LDAEP was higher in patients with atypical depression than in those with non-atypical depression. A recent small study $(n=16)$ found that the BSS score did not differ significantly between small- and large-LDAEP groups based on a dichotomized stratification at the median LDAEP, whereas the score on the Barratt Impulsiveness Scale (BIS) did differ significantly between the two groups. ${ }^{24}$

\section{LDAEP AND HISTORY OF SUICIDE ATTEMPTS IN PATIENTS WITH MDD}

Some investigators initially reported that a small intensity dependence of somatosensory evoked potentials was related to suicidal attempts in patients with MDD. ${ }^{25}$ These results were replicated for the intensity dependence of auditory-evoked potentials. ${ }^{21}$ That study found the intensity dependence to be smaller in patients with a history of suicide attempts $(n=7)$ than in those with no suicide history $(n=15)$. However, these results were consistent with most previous studies finding low serotonergic activity to be associated with suicide attempts (using different methods, such as postmortem CSF measurements). ${ }^{2}$ This discrepancy might be attributable to the suicide attempts of these patients having occurred in the past and hence being unrelated to the current medical contact, and that the relationship between low serotonergic activity and suicide attempts was mainly found during the acute suicidal state.

A study involving Taiwan patients produced contradictory results, ${ }^{26}$ finding that the LDAEP was larger in depressed patients who had attempted suicide $(n=16)$ than in those who had not $(n=50)$. These results were replicated in another study also involving subjects divided into those who had attempted suicide $(n=17)$ and those who had not $(n=21) .{ }^{19}$ The LDAEP was calculated by measuring auditory event-related potentials (ERP), and differed significantly between the two groups. Depressive patients with a history of suicide attempts seem to be characterized by a large LDAEP, indicating low serotonergic activity. Another study found that the LDAEP did not differ significantly between suicide attempts $(n=11)$ and no suicide attempts $(n=130) .{ }^{27}$ However, that study was not originally designed to investigate the relationship between the LDAEP and suicide attempts, and the group with a suicide-attempts history was much smaller than the group without such a history.

The above-described results indicate that the relationship between suicide attempts and the LDAEP is still controversial. A key feature of all of the studies is that they have involved relatively small samples. In addition, almost all subjects included in the suicide-attempters group were not acute suicide attempters, instead having a history of suicide attempts. Thus, a more precise evaluation requires the application of different methodology. For example, the LDAEP should be measured immediately after the suicide attempts of patients with MDD, rather than simply assessing the history of suicide attempts. One recent study with a different design was performed by Uhl and colleagues, ${ }^{20}$ in which the LDAEP was measured at about 2, 5, 9, and 16 days after attempted suicide or a suicide attempt. The LDAEP was significantly larger on day 9 than on days 2 and 16, and tended to be larger on day 9 than on day 5. Instability of central serotonergic function is suggested to result in reduced serotonergic activity at about 1 week after a suicide attempt. Thus, serotonergic dysfunction in suicidality seems to be more complicated than was originally thought. However, that study had several limitations, including 1) the 
smallness of the sample, 2) the noninclusion of nonsuicidal controls, and 3) no control of medications such as antidepressants and antipsychotics. Another study investigated the relationship between the LDAEP and self-injurious behavior. ${ }^{28}$ The LDAEP was measured in 41 men, who then completed the Suicidal Behaviors Questionnaire (SBQ) and the Deliberate Self-Harm Inventory (DSHI). The LDAEP was positively correlated with scores on the SBQ and DSHI. In addition, in subjects with a history of actual suicide attempts, a larger LDAEP was related to higher scores on measures of self-injurious behaviors across the spectrum of lethality. However, that study also had significantly limitations: 1) the sample was relatively small (as is the case for most ERP studies), 2) the study exclusively relied on self-report behavioral measures of lifetime SIB, and 3) only men were included.

Juckel and Hegerl ${ }^{21}$ found that the LDAEP of the tangential dipole was smaller in alcoholic patients with a history of suicide attempts $(\mathrm{n}=10)$ than in those without such a history $(\mathrm{n}=$ 15). However, the LDAEP of the radial dipole did not differ significantly between two groups. Park and $\mathrm{Lee}^{29}$ evaluated the LDAEP in patients with acute major depressive episode. They divided 61 patients into 2 groups based on whether or not they achieved a positive score on the Mood Disorder Questionnaire (MDQ). The LDAEP was measured before beginning medication with serotonergic agents, and it was found that the LDAEP did not differ significantly between MDD patients with and without bipolarity. However, the BSS score (which reflects impulsivity) was higher in the MDD patients with bipolarity, and applying binary logistic regression analysis to bipolarity revealed that the relationship between the total MDQ score and the BIS or BSS score was also significant. It is therefore possible that suicidality in the presence of a mood disorder depends on impulsivity and bipolarity as well as the serotonin level. ${ }^{30}$

\section{DISCUSSION AND FUTURE DIRECTIONS}

Serotonergic dysfunction in suicidality seems to be more complicated than was originally thought. Studies of suicide based on the LDAEP have produced controversial results, but it is possible that these discrepancies are due to differences in study designs and the smallness of the included samples. For example, some studies have evaluated suicide ideation and the LDAEP, while others have evaluated suicide attempts and the LDAEP. Furthermore, some of the latter studies enrolled acute suicide attempters, while others enrolled those with a history of previous suicide attempts, irrespective of whether these were acute or chronic. Thus, a robust study design is needed in future studies, such as by evaluating the LDAEP immediately after a suicide attempt rather than in those with a history of suicide attempts and suicide ideation in order to reduce bias. Moreover, genuine suicide attempt, self-injurious behaviors, and faked suicide attempt need to be discriminated in the future. Medication can also affect the LDAEP, and so ERP has to be measured in a drug-naive state. Another consideration is the ERP methodology. All of studies reported in this article used only the scalp N1/P2 LDAEP as measured at $\mathrm{Cz}$, and each N1 LDAEP and P2 LDAEP need to be analyzed in order to provided detail outcomes in the future. In addition to these analyses, source analysis such as standardized lowresolution brain electromagnetic tomography (sLORETA) should be performed in large samples in future studies because some studies have produced different findings according to the use of scalp analysis or source analysis.

\section{Acknowledgments}

This study was supported by a grant from National Research Foundation of Korea (NRF), funded by Ministry of Education (NRF-2014R1A1A2A 10059345). The authors would like to thank Jo BW for her assistance with data collection.

\section{REFERENCES}

1. Maris RW. Suicide. Lancet 2002;360:319-326.

2. Mann JJ. The neurobiology of suicide. Nat Med 1998;4:25-30.

3. Bourne HR, Bunney WE Jr, Colburn RW, Davis JM, Davis JN, Shaw DM, et al. Noradrenaline, 5-hydroxytryptamine, and 5-hydroxyindoleacetic acid in hindbrains of suicidal patients. Lancet 1968;2:805-808.

4. Shaw DM, Camps FE, Eccleston EG. 5-Hydroxytryptamine in the hindbrain of depressive suicides. Br J Psychiatry 1967;113:1407-1411.

5. Mann JJ, Huang YY, Underwood MD, Kassir SA, Oppenheim S, Kelly $\mathrm{TM}$, et al. A serotonin transporter gene promoter polymorphism (5-HTTLPR) and prefrontal cortical binding in major depression and suicide. Arch Gen Psychiatry 2000;57:729-738.

6. Pandey GN, Dwivedi Y, Rizavi HS, Ren X, Pandey SC, Pesold C, et al. Higher expression of serotonin 5-HT(2A) receptors in the postmortem brains of teenage suicide victims. Am J Psychiatry 2002;159:419-429.

7. Arora RC, Meltzer HY. Serotonergic measures in the brains of suicide victims: 5-HT2 binding sites in the frontal cortex of suicide victims and control subjects. Am J Psychiatry 1989;146:730-736.

8. Lee HY, Hong JP, Hwang JA, Lee HJ, Yoon HK, Lee BH, et al. Possible association between serotonin transporter gene polymorphism and suicide behavior in major depressive disorder. Psychiatry Investig 2015; 12:136-141.

9. Antypa N, Serretti A, Rujescu D. Serotonergic genes and suicide: a systematic review. Eur Neuropsychopharmacol 2013;23:1125-1142.

10. Correa H, Duval F, Mokrani M, Bailey P, Tremeau F, Staner L, et al. Prolactin response to D-fenfluramine and suicidal behavior in depressed patients. Psychiatry Res 2000;93:189-199.

11. Juckel G, Molnar M, Hegerl U, Csepe V, Karmos G. Auditory-evoked potentials as indicator of brain serotonergic activity--first evidence in behaving cats. Biol Psychiatry 1997;41:1181-1195.

12. Hegerl U, Juckel G. Intensity dependence of auditory evoked potentials as an indicator of central serotonergic neurotransmission: a new hypothesis. Biol Psychiatry 1993;33:173-187.

13. Buchsbaum M, Silverman J. Stimulus intensity control and the cortical evoked response. Psychosom Med 1968;30:12-22.

14. Juckel G, Hegerl U, Molnar M, Csepe V, Karmos G. Auditory evoked potentials reflect serotonergic neuronal activity--a study in behaving cats administered drugs acting on 5-HT1A autoreceptors in the dorsal raphe nucleus. Neuropsychopharmacology 1999;21:710-716. 
15. Hegerl U, Gallinat J, Juckel G. Event-related potentials. Do they reflect central serotonergic neurotransmission and do they predict clinical response to serotonin agonists? J Affect Disord 2001;62:93-100.

16. Strobel A, Debener S, Schmidt D, Hunnerkopf R, Lesch KP, Brocke B. Allelic variation in serotonin transporter function associated with the intensity dependence of the auditory evoked potential. Am J Med Genet B Neuropsychiatr Genet 2003;118B:41-47.

17. Park YM, Lee SH, Park EJ. Usefulness of LDAEP to predict tolerability to SSRIs in major depressive disorder: a case report. Psychiatry Investig 2012;9:80-82.

18. Hegerl U, Bottlender R, Gallinat J, Kuss HJ, Ackenheil M, Moller HJ. The serotonin syndrome scale: first results on validity. Eur Arch Psychiatry Clin Neurosci 1998;248:96-103.

19. Kim DH, Park YM. The association between suicidality and serotonergic dysfunction in depressed patients. J Affect Disord 2013;148:72-76.

20. Uhl I, Illes F, Grassnickel V, Echterhoff S, Norra C, Juckel G. Loudness dependence of auditory evoked potentials (LDAEP) in clinical monitoring of suicidal patients with major depression: a pilot study. Eur Arch Psychiatry Clin Neurosci 2012;262:487-492.

21. Juckel G, Hegerl U. Evoked potentials, serotonin, and suicidality. Pharmacopsychiatry 1994;27(Suppl 1):27-29.

22. Park YM, Lee BH, Lee SH. The association between serum lipid levels, suicide ideation, and central serotonergic activity in patients with major depressive disorder. J Affect Disord 2014;159:62-65.

23. Lee SH, Park YC, Yoon S, Kim JI, Hahn SW. Clinical implications of loudness dependence of auditory evoked potentials in patients with atypical depression. Prog Neuropsychopharmacol Biol Psychiatry 2014; 54:7-12.

24. Park YM, Lee BH, Um TH, Kim S. Serum BDNF levels in relation to illness severity, suicide attempts, and central serotonin activity in patients with major depressive disorder: a pilot study. PLoS One 2014;9: e91061.

25. Agren H, Osterberg B, Franzen O. Depression and somatosensory evoked potentials: II. Correlations between SEP and depressive phenomenology. Biol Psychiatry 1983;18:651-659.

26. Chen TJ, Yu YW, Chen MC, Wang SY, Tsai SJ, Lee TW. Serotonin dysfunction and suicide attempts in major depressives: an auditory eventrelated potential study. Neuropsychobiology 2005;52:28-36.

27. Min JA, Lee SH, Lee SY, Chae JH, Lee CU, Park YM, et al. Clinical characteristics associated with different strengths of loudness dependence of auditory evoked potentials (LDAEP) in major depressive disorder. Psychiatry Res 2012;200:374-381.

28. Marsic A, Berman ME, Barry TD, McCloskey MS. The relationship between intentional self-injurious behavior and the loudness dependence of auditory evoked potential in research volunteers. J Clin Psychol 2015;71:250-257.

29. Park YM, Lee SH. Can the loudness dependence of auditory evoked potentials and suicidality be used to differentiate between depressive patients with and without bipolarity. Psychiatry Investig 2013;10:143-147.

30. Park YM, Lee SH. Clinical Usefulness of Loudness Dependence of Auditory Evoked Potentials (LDAEP) in patients with bipolar disorder. Psychiatry Investig 2013;10:233-237. 